\title{
Agresión y Resentimiento en los Estudiantes Secundarios*
}

\section{Aggression and Resentment in High-school students}

\author{
Francisco Leal \\ Mónica Meneses \\ Andrés Alarcón \\ Vesna Karmelic ${ }^{* *}$
}

\begin{abstract}
Resumen
Se explora la relación entre conductas agresivas en contextos escolares de vulnerabilidad social, y resentimiento, estado de ánimo relativamente permanente que puede surgir con mayor probabilidad en contextos de discriminación, exclusión y marginación social. Se midió el resentimiento con una escala específica y se clasificó la gravedad de las conductas agresivas a partir de las observaciones en el registro escolar individual, en 270 estudiantes de segundo y tercero medio de un liceo de alta vulnerabilidad social. Los alumnos que presentaban conductas agresivas graves mostraron significativamente más resentimiento que el resto de la muestra. Se propone que las conductas agresivas podrían ser comprendidas, al menos parcialmente, como reacción a la violencia social evidente en estos liceos.
\end{abstract}

Palabras clave: resentimiento-agresividad-estudiantes secundarios.

\begin{abstract}
The relationship between aggressive behavior and resentment in vulnerable school contexts was studied, a relatively permanent emotional state that arises more probably in contexts of social discrimination or exclusion. Resentment was assessed with a specific scale and the severity of aggressive behavior was established from individual school records in 270 students attending grades 10 to 11 in a school within a context of high social vulnerability. The students with higher degrees of aggressive behavior also showed significantly more resentment than the other students. The suggestion is that aggressive behavior could be understood, at least in part, as reactive to the social violence present in these schools.
\end{abstract}

Key words: resentment-aggressiveness-secondary school students.

* Este artículo corresponde a un trabajo realizado como parte de un programa de investigación desarrollado a través de actividades de graduación de la Carrera de Psicología de la Universidad de Tarapacá.

** Psicólogos, Depto. de Filosofía y Psicología, Universidad de Tarapacá. email fleal@uta.cl 
La violencia y los comportamientos disruptivos en la escuela son cada vez más frecuentes, y han llegado a constituirse en un problema serio. Aunque se presentan en todo tipo de establecimientos, tienden a concentrarse en las escuelas -particularmente los liceos- de sectores populares (Bravo, 2000; Rodríguez et al., 2004). La agresividad por sí misma, sin embargo, no es necesariamente desadaptativa, dado que ciertos comportamientos agresivos son necesarios para adaptarse, para enfrentar desafíos y para defenderse de los ataques; la agresividad se torna problemática y se transforma en violencia cuando fallan los mecanismos que la regulan, como señala Barudy (2000). Este autor propone un modelo en que la violencia humana puede ser explicada en tres niveles: en el nivel de los trastornos de apego o vinculación personal, en el de los trastornos de la representación sobre la naturaleza de las relaciones interpersonales o sistemas de creencias, y en el de los contextos que interfieren en el desarrollo integral de todos los miembros de una sociedad, como la pobreza y la exclusión. Es probable que los comportamientos agresivos observados en estos liceos socialmente vulnerables correspondan principalmente a este último nivel, ya que una proporción importante de sus alumnos provienen de familias pobres o marginadas, y han sufrido habitualmente formas de exclusión social y fracaso escolar (Bellei 2000; Weinstein 2001).

Echeverría (1996) plantea que el resentimiento es un estado emocional que se construye en la narrativa personal y que representa la interpretación de haber sido víctima de un acto injusto por parte de otro $u$ otros, frente al cual se rebela. Este estado se manifiesta en la forma de una hostilidad generalizada y manifestada indirectamente en distintas formas de agresión difusa (Delgado 1938).

En este trabajo, intentamos establecer si la conducta disruptiva y los comportamientos agresivos observados en un liceo de alta vulnerabilidad socioeducativa -un liceo que presenta indicadores socioeconómicos muy bajos en sus familias y altos indicadores de fracaso y deserción escolar, por lo que una proporción importante de sus alumnos ha sufrido situaciones de carencia y exclusión social- pueden ser explicados parcialmente por el resentimiento que podría asociarse a tales carencias y exclusiones.

\section{Disrupción, agresión y violencia}

Es necesario distinguir entre violencia propiamente tal, y comportamientos disruptivos en la escuela. Los comportamientos disruptivos son comportamientos consistentes en actitudes o acciones destinadas a la transgresión de normas explicitadas, que se encuentran reguladas y conocidas por todos los involucrados dentro del ambiente educativo; tienden a desestabilizar la vida cotidiana en el proceso de enseñanza, lo cual puede variar de menor a mayor intensidad, incluyendo acciones agresivas que pueden estar dirigidas tanto a objetos como a personas, y que pueden ser verbales, físicas y/o emocionales, pero que no provocan un daño específico a alguien (Fernández 1998). Por tanto, aquellas actidudes y / o comportamientos que provocan un estado de inquietud dentro del aula corresponden a una "disrupción". Tattum (1989) señala que, en el lenguaje de los profesores, ésta se interpreta como un conglomerado de conductas inapropiadas, tales como falta de cooperación y mala educación, insolencia, desobediencia, provocación y agresión, hostilidad y abuso, impertinencia, amenazas, etc. También se pueden mostrar como estrategias verbales, tales como pedir que se explique lo ya explicado con ánimo de retrasar la tarea, hacer preguntas absurdas, reaccionar desproporcionadamente a una instrucción exagerando su cumplimiento, vestir ropa $u$ objetos grotescos, demostrar expresiones desmesuradas de aburrimiento, etc. Aunque estos comportamientos pueden ser presentados por niños con síndrome de déficit atencional o hiperkinesia, este cuadro debe diferenciarse 
claramente, ya que responde a causas diferentes y los comportamientos disruptivos ocasionados por niños con el mismo no necesariamente son intencionales o transgresores (García y Magaz, 2000).

La disrupción en las aulas constituye la preocupación más directa y la fuente de malestar más importante dentro del contexto educativo, ya que impide al profesor desarrollar su tarea de instruir, o formulado a la inversa, interfiere con el aprendizaje de los alumnos debido a los desórdenes, indisciplina, escasa motivación y apatía en el proceso de enseñanza dentro del aula que se generan como consecuencia (Fernández, 1998).

La disrupción proporciona un campo apropiado para la aparición de conflictos a partir de los cuales se va forjando un malestar creciente, que comenzaría por una actitud negativa por parte de algunos alumnos, expresada en problemas de conductas, baja autoestima y claros problemas de "saber estar", o falta de habilidades sociales de escucha y respeto por normas convencionales. Tal situación conflictiva de no acatamiento a las normas, se puede observar dramáticamente cada vez que los alumnos son coercionados, sancionados o expulsados por el medio escolar, lo que redunda en un aumento de dichos comportamientos disruptivos que termina provocando una actitud negativa, del campo, deviniendo en relaciones interpersonales tirantes entre profesor y alumnos, provocando un ambiente de inquietud y malestar, dificultando la convivencia, la realización de las tareas propias de la escuela y tensionando el ambiente en general (Fernández 1998).

La violencia implica un daño físico, verbal o psicológico específico a algún miembro de la comunidad, e incluye comportamientos como los abusos, las agresiones directas, físicas o verbales, y el vandalismo (Fernández, 1998). Aunque este tipo de comportamientos es más impactante, y se hace público con mayor facilidad, es el comportamiento disruptivo el que ocurre con mayor frecuencia en la escuela, y constituye preocupación más constante de los profesores. En este trabajo, abordaremos ambos tipos de comportamientos, a los que nos referiremos genéricamente como comportamientos agresivos.

\section{Resentimiento}

El resentimiento, por el carácter principalmente moral que asume, ha sido tratado principalmente por filósofos. Nietzsche (1993) presenta el amor cristiano como el mejor ejemplo del resentimiento, al surgir desde la impotencia para manifestar el odio o realizar la venganza: al quedar vedada la posibilidad de actuar para expresar tales sentimientos por el mandato cristiano, éstos serían reprimidos dando lugar al resentimiento, el que, al ser sublimado, generaría el movimiento creador que culminaría en el amor cristiano. Independientemente de que estemos de acuerdo o no con el ejemplo de Nietzsche, éste destaca uno de los elementos centrales presentes en el resentimiento: la imposibilidad de expresar activamente los sentimientos negativos y agresivos hacia otras personas. Coincidiendo con esto, May (1976) señala desde la Psicología que el resentimiento es la apariencia del odio en la vida urbana y civilizada, y Delgado, al señalar desde la Psiquiatría las características distintivas del resentimiento, aludía muy tempranamente, entre otras, a que se trata de una hostilidad sin objeto individual exclusivo y en que la movilización no se actualiza de manera adecuada o directa, sino indirectamente, con transposiciones, agresividad difusa, envidia velada, rencor sordo, etc. (Delgado 1938).

Scheller (1938) es más explícito y analítico que Nietzsche. Coincide en que el resentimiento se produce por la represión sistemática de emociones negativas, y la describe como una reacción emocional recurrente, que se vive y revive repetidamente y se aleja de la esfera expresiva. El resentimiento se vive característicamente con una cualidad negativa u hostil, y se constituye en fuente de juicios valorativos distorsionados. 
En su descripción psicofenomenológica de la personalidad, Lersch (1971) incluye al resentimiento como la expresión pasiva del afán vindicativo que surge cuando la expresión activa -la venganza- no es posible. El afán vindicativo, a su vez, surge cuando hay un daño o una insatisfacción de las necesidades vinculadas a las vivencias pulsionales del yo -conservación, egoísmo, poder, estima y autoestima.

Buss (1996) lo define como un sentimiento de cólera a partir de un maltrato real o ficticio, verbalizado como disgusto, gemidos, queja y exigencia. Esta definición es interesante, ya que enfatiza dos ideas relevantes: la de maltrato, idea que coincide con el planteamiento de Lersch, y la de percepción subjetiva del mismo, lo que apunta a que una persona puede manifestar resentimiento a partir de su percepción subjetiva de haber sido maltratada, independientemente de si los hechos reales que la originan constituyen o no efectivamente un maltrato.

Coincidiendo con estos planteamientos, Echeverría (1996) describe al resentimiento como uno de los cuatro estados de ánimo básicos, junto a la resignación, la aceptación y la ambición. Estos estados surgen como consecuencia de la actitud que la persona asume frente a los juicios que hace de su situación. De acuerdo a Echeverría, hay dos tipos de juicios posibles: de facticidad y de posibilidad. Un juicio de facticidad es la adscripción que hace la persona de inmodificabilidad de una situación. Es decir, una persona hace un juicio de facticidad cuando cree que alguna situación específica que la afecta es inmutable. Cuando se hace un juicio de esta naturaleza, la persona puede aceptarlo o rechazarlo; el resentimiento es el estado de ánimo resultante de rechazar una facticidad que es percibida como ataque $u$ ofensa hacia la propia persona.

Así, según Echeverría, el resentimiento como estado emocional se construye en la narrativa personal como una conversación subyacente que representa la interpretación de haber sido víctima de un acto injusto por parte de otro u otros, frente al cual la persona se rebela. Lo injusto deviene de la idea de haber tenido derecho moral a obtener o hacer algo que, no obstante ese derecho, le fue negado (León, Romero, Novara y Quesada 1988); esta negación es la que es percibida como injusta, como un agravio hacia la persona. En esta interpretación, alguien aparece como culpable por impedir que se obtuviera lo que se merece; este alguien podría ser una persona, un grupo o una clase de personas, una institución, la sociedad, el mundo o la vida en general (Echeverría 1996; Buss 1996).

Para Echeverría, el resentimiento se nutre de las promesas y las expectativas incumplidas. Ambas confieren el derecho de esperar un determinado comportamiento de otros. Cuando alguien promete, confiere a la otra persona el derecho de esperar legítimamente que la promesa se cumpla. Sin embargo, hay ciertas convenciones sociales de comportamiento, propios de cada comunidad, que son independientes de las promesas que cada individuo formule explícitamente, y que generan legítimas expectativas. No tiene sentido, por ejemplo, afirmar: "Yo nunca prometí hacerme responsable de mis hijos", puesto que hay una convención social que hace innecesaria la formulación de dicha promesa para generar la expectativa de cumplimiento. Si las promesas o expectativas no se cumplen, y este incumplimiento es percibido como permanente, es altamente probable que surja el resentimiento, como una invocación de justicia frente a la injusticia del derecho no respetado.

Cabe señalar que, tal como apuntan Buss (1996) y Echeverría (1996), esta percepción de injusticia puede tener sus bases tanto en la ocurrencia de una ofensa o ataque manifiesto e intencional, como en la vivencia subjetiva de haber sido ofendido o atacado a partir de un acto carente de tal intencionalidad, pero al cual el sujeto resentido se la atribuye. Esto puede ocurrir especialmente cuan- 
do ya se ha experimentado resentimiento, ya que se comienza a percibir selectivamente aquellas situaciones que confirman la idea de haber sido víctima de acciones injustas, aumentando la posibilidad de atribuir esta intencionalidad a los actos de los demás. Por otra parte, generalmente no es un solo o único hecho el que gatilla el resentimiento; en la mayoría de los casos se trata de una acumulación de hechos experimentados subjetivamente como injustos. Así, puede resultar muy predisponente al resentimiento un ambiente en el cual a diario se presentan injusticias, postergaciones o maltratos (Castillo 1982; León et al. 1988; Scheller 1938). De este modo, pequeños enojos o frustraciones adquieren importancia al acumularse, ya que por lo general tienen un efecto aditivo (Nathan y Harris 1989), favoreciéndose el resentimiento cuando se suceden situaciones de maltrato o postergación y/o cuando el sujeto va selectivamente otorgando mayor relevancia a aquellas que considera injustas (Titze 1983).

Las frustraciones que generalmente llevan al resentimiento tienen que ver con barreras y obstáculos que se interponen en el logro de aspiraciones que la persona tiene, sean de bienestar, de posesión, de poder o de estimación, generalmente aquellos de carácter más permanente (Lersch 1976; Buss 1996). También juegan un rol las frustraciones producto de la omisión de recompensas, lo que deriva del incumplimiento de promesas y expectativas (Nathan y Harris 1989; Buss 1996; Jones y Gerard 1980).

Finalmente, el resentimiento también puede surgir, como se ha señalado ya, de la imposibilidad o impotencia de actualizar o manifestar expresiva y abiertamente los sentimientos negativos como el odio, la venganza y la envidia (Scheller 1938). En cualquier caso, para que surja el resentimiento, la sola presencia de frustración o percepción de injusticia no es suficiente; es necesario que el impulso inmediato que surge para responder a la ofensa o ataque, o para reparar la injusticia, sea obstruido o imposible, ya que lo adecuado en tal caso sería la manifestación directa de tales sentimientos. El resentimiento es el resultado de esta coacción. De acuerdo a Sullivan (1964), el resentimiento puede surgir como resultado de la consideración que hace la persona acerca de las consecuencias que puede tener para él la expresión de sus sentimientos negativos hacia alguien que tiene una situación privilegiada de poder, o incluso, como resultado de amenazas violentas y represoras ejercidas por alguien que hacen que la persona se sienta incapaz de defenderse. Cualquiera sea el origen de esta imposibilidad de manifestar los sentimientos negativos, el efecto es que la persona ejerce autocontrol evitando y posponiendo la expresión de la emoción negativa (Nathan y Harris 1989) y eventualmente desplazándola para otro momento y situación más apropiada; cuando esto se hace recurrente, se transforma en resentimiento (Scheller 1938).

De este modo, el resentimiento es un estado de ánimo recurrente, que surge al ser o considerarse imposible la manifestación abierta de los sentimientos negativos hostiles surgidos por el rechazo de una situación o facticidad considerada como ofensiva o injusta, que produce daño o menoscabo a su persona o sus aspiraciones. Este estado de ánimo se expresa en disposiciones de acción indirectas o desplazadas. Tomando como base y ampliando la definición que hicieran León et al. (1988), asumimos como definición provisional de resentimiento la siguiente: estado de ánimo relativamente permanente que surge del juicio de haber sido maltratado o postergado injustamente en el logro de derechos o expectativas que se considera legítimos, que se manifiesta en actitudes hostiles hacia las cosas, las personas, la vida, el mundo o sí mismo.

Operativamente, el resentimiento involucra: el revivir constante de sentimientos o emociones del pasado, relacionados con situaciones de abuso, postergación o injusticia, reales o imaginarias; autopercepción de 
ser víctima; creencia de haber obtenido menos de lo merecido; culpabilización de otros por su postergación; sentimiento de impotencia; retención o expresión indirecta de sentimientos negativos; agresividad latente; juicios críticos hacia el mundo externo; promesa y/o búsqueda de ocasiones que puedan dar lugar a actos de venganza internos; hostilidad.

Aunque algunas personas parecen más predispuestas que otras al resentimiento por razones personales (Scheller 1938), por nuestro ámbito de interés nos preocupan mucho más las razones sociales que contribuyen al mismo. Nos referimos a aquellas situaciones características y frecuentes en nuestra sociedad que se constituyen en fuente de frustraciones y de incumplimiento de derechos y expectativas, y que devienen en gran parte de una característica esencial de nuestra sociedad: su jerarquización, asociada a desigual distribución de recursos y poder. Como afirma Echeverría (1996), la relación entre distribución de poder y resentimiento es crítica. En una sociedad más igualitaria habría menos posibilidad de resentimiento, aunque, como advierte Scheller (1938) la máxima carga de resentimiento correspondería a aquella sociedad en que los derechos políticos y la igualdad social están presentes a nivel discursivo, pero coexiste con diferencias muy notables en el poder, la riqueza y la educación efectivos.

Éste es el caso de la mayor parte de nuestras sociedades latinoamericanas, que incorporan la igualdad en su discurso oficial y mediático, pero en las cuales las inequidades son también evidentes (Blanco y Cusato, s/ d); de este modo, es fácil advertir cómo en nuestros sistemas de convivencia se presentan frecuente y sistemáticamente situaciones de atropello y abuso que pueden generar resentimiento, como el maltrato familiar, la discriminación étnica y social y, sobre todo, las condiciones de pobreza de grandes sectores de la población, en contraste con la manifiesta riqueza de algunos y con el bienestar fácil proclamado majaderamente a través de los medios de comunicación.
Maturana (1992) señala que nuestro sistema de convivencia es un sistema jerárquico de poder que se constituye sobre la base de las emociones que sustentan la autonegación y la negación del otro, y la aceptación del sometimiento propio o del otro en una dinámica de orden y obediencia, surgidas en un proceso de socialización que es fundamentalmente limitador de las emociones. Por su parte, Echeverría (1996) señala que encontrarse en una situación carente de poder es motivo suficiente para no manifestar los sentimientos de rabia o descontento, posibilitando el surgimiento del resentimiento contra quien o quienes representan el abuso de poder. La violencia social como fenómeno se sustenta en esta situación de abuso, manifestándose en la diferenciación de unas personas por otras, en relaciones discriminatorias basadas en características como sexo, raza, edad, formas de vestir, nivel de ingresos o nivel cultural (Troncoso, 1998).

Quienes son víctimas con mayor frecuencia de estos abusos son los niños, las mujeres, los jóvenes, los pobres y las minorías étnicas, como categorías genéricas, lo que no es casual en nuestra estructura social y económica capitalista, en la que algunos concentran el poder económico, político y cultural. De hecho, como señala Castillo (1982), los grupos marginados no son grupos abandonados en el desarrollo de un país, sino un producto directo del mismo desarrollo; la marginalidad es la forma de pertenencia e incorporación a la sociedad, por lo que esta distribución injusta es una facticidad del sistema. Los niños y jóvenes, en particular, se encuentran expuestos a situaciones de agravio social: el contexto escolar, por ejemplo, tiende a poner en desventaja a los niños y jóvenes de niveles socioeconómicos bajos (Castillo, 1982; Weinstein, 2001); el sistema educativo es contradictorio, elitista e injusto. La escuela quiere niños y jóvenes limpios, bien vestidos, ordenados, con buen dominio del lenguaje y "buenas costumbres", y poco de esto corresponde a los niños y jóvenes pobres. 
Esta situación no puede sino producir un sentimiento de injusticia, que muchas veces, por temor a un mayor rechazo o simplemente por no percibir las desigualdades que le dan origen, no se orienta hacia las fuentes de la frustración, sino que comienzan a referirse a la vida, el mundo o la propia existencia (Troncoso, 1998; González, 1991). El autoritarismo, las descalificaciones constantes, las amenazas de castigo, hacen sentir al joven que no puede o no debe reaccionar frente a las fuentes de sus tensiones, acumulando sentimientos negativos que luego descarga en tiempos, espacios y personas que no se relacionan directamente con su problema (Castillo, 1982).

\section{Hipótesis}

El problema de la investigación fue determinar si los alumnos que presentan distintos niveles de comportamientos agresivos difieren también en sus niveles de resentimiento; en otras palabras, si el resentimiento podría ser una variable explicativa de los comportamientos agresivos observados en el contexto escolar. Nuestra hipótesis fue que los alumnos que exhiben más y más graves comportamientos agresivos son también los que muestran mayores niveles de resentimiento, y viceversa.

\section{Diseño y muestra}

Utilizamos un diseño expost-facto retrospectivo (León y Montero, 1997), y decidimos conformar los grupos a partir de la variable comportamiento agresivo. La muestra fueron 270 alumnos, de ambos sexos, que asistían a $2^{\circ}$ y $3^{\circ}$ de enseñanza media en un liceo considerado de alta vulnerabilidad socioeducativa, de la ciudad de Arica.

\section{Procedimiento e instrumentos}

La presencia de comportamientos agresivos se determinó a partir del examen del "libro de clases", en que se registra anotacio- nes para cada alumno. Según la cantidad y el tipo de anotaciones registradas, cada alumno fue asignado a uno de cuatro grupos:

0 , ausencia de comportamientos agresivos: no registra anotaciones por conductas disruptivas o violentas.

1, presencia leve de comportamientos agresivos: registra hasta un máximo de cuatro anotaciones por conductas disruptivas que no implican violencia, es decir, no conllevan daños o efectos negativos para sí mismo, para otros ni para la propiedad, tales como provocar desorden, presentarse sin uniforme o incumplimiento reiterado de tareas.

2 , presencia moderada de comportamientos agresivos: registra entre cinco y siete anotaciones por conductas agresivas, entre las que puede incluirse algunas por comportamientos violentos, es decir, que implican riesgo o daño importante para sí mismo, para otros o para la propiedad, como fugarse de la escuela, pelear, jugar con fuego o provocar destrozos.

3, presencia grave de comportamientos agresivos: registra ocho o más anotaciones por conductas agresivas, entre las que se cuentan varias por comportamientos violentos, es decir, que implican importante riesgo o daño, como maltrato a compañeros, vandalismo y provocar incendios. La mayor parte de los alumnos de este grupo consignaban anotaciones que establecían la condicionalidad de su permanencia en el liceo.

El resentimiento se evaluó con una escala desarrollada específicamente para evaluar esta variable en jóvenes chilenos (Leal, Sepúlveda, Vargas, Braniff e Irrazával 2003), que se presenta como una escala de actitudes para la vida y consta de cuarenta ítemes dicotómicos que se responden con acuerdo o desacuerdo. Cada ítem es puntuado con los valores 0 y 1 , variando entonces el puntaje posible entre 0 y 40 ; el mayor puntaje indica 
una mayor presencia de indicadores de resentimiento. La confiabilidad por consistencia interna de esta escala es aceptable (Alpha de Cronbach $=0,83$ ), presenta buena validez para discriminar entre grupos establecidos por criterio de expertos ( $t$ de Student $=6,32$, $\mathrm{gl}=49, \mathrm{p}=0.000)$, y las puntuaciones no presentan diferencias por sexo. Esta escala fue aplicada a los alumnos en horario de clases, aunque en una sala distinta de la habitual y en grupos de cinco alumnos, por uno de los investigadores.

\section{Resultados}

En la Tabla 1 se aprecia que las medias en la escala de resentimiento muestran una clara tendencia a aumentar según aumenta la presencia y gravedad de los comportamientos agresivos. A pesar de las diferencias en el tamaño de los grupos, no hay diferencias significativas entre sus varianzas (Estadístico de Levene $=0,693$, gl1 $=3, \mathrm{gl} 2=266$, $\mathrm{p}=0.557$ ), por lo que se realizó directamente un análisis de varianza (ANOVA) cuyos resultados, que se muestran en la Tabla 2, confirman la significación de las diferencias entre los grupos.

Se realizó una prueba post-hoc de comparaciones múltiples, escogiéndose la de Scheffé por su insensibilidad a las diferencias de tamaño entre los grupos (Ferrán 1996). Este análisis, cuyos resultados se presentan en la Tabla 3, muestra varias diferencias significativas, siendo lo más relevante que el grupo que exhibe comportamientos agresivos graves presenta puntajes significativamente mayores que los otros tres grupos en la escala de resentimiento; es decir, este grupo presenta significativamente mayor puntaje en la escala de resentimiento que cualquiera de los otros tres. El grupo que no presenta comportamientos agresivos se diferencia significativamente del grupo que los presenta moderadamente, pero no del grupo que los presenta levemente. Tampoco hay diferencia significativa entre los grupos de comportamientos agresivos leve y moderado.

\section{Discusión}

Los resultados confirman claramente la hipótesis de que la cantidad y gravedad de comportamientos agresivos en la escuela se asocian positivamente con el nivel de resentimiento. En particular, la presencia grave de comportamientos agresivos, que implica reiterados comportamientos violentos, se asocia a niveles significativamente más altos de resentimiento que menores niveles de comportamientos agresivos.

Por el diseño no explicativo del estudio, no puede asignarse un rol causal al resentimiento en relación a los comportamientos agresivos a partir de estos resultados. Sin embargo, dados los antecedentes teóricos, es razonable proponer que las condiciones de marginación y exclusión social, que predisponen a experiencias escolares de fracaso, den lugar, en el jerarquizado y discriminador contexto escolar (Magendzo, 2000), a mayores niveles de resentimiento, los que se expresarían a través de estos comportamientos agresivos.

A partir de esta razonable suposición, se desprenden dos conclusiones relevantes. La primera es la necesidad de explorar la relación entre resentimiento y comportamientos agresivos en el medio escolar con un diseño que permita establecer su eventual causalidad. O, tal vez más importante, explorar esta relación a nivel de significaciones en los propios jóvenes, sea a nivel colectivo o individual. Justamente por su carácter desplazado, resulta difícil para los mismos jóvenes reconocer la fuente de sus comportamientos agresivos, más allá de su objeto inmediato. Abrir para ellos y para quienes los rodean la posibilidad de explorar estas relaciones supondría una nueva opción de manejar las cosas de maneras diferentes.

En el mismo sentido, la segunda conclusión relevante es la urgente necesidad de incorporar la enorme violencia social manifiesta en nuestra sociedad al análisis de la 
realidad juvenil, en particular a la de las situaciones escolares. La agresión que se les atribuye a los jóvenes no surge como un fenómeno aislado; estos resultados indican que es mediada por el resentimiento, y éste, aunque es una variable individual, es probablemente producto de la manifiesta injusticia social a que se encuentran expuestos los jóvenes de liceos más vulnerables, y de su también manifiesta impotencia al respecto. No se avanzará mucho buscando soluciones para la violencia en las escuelas, mientras no se comprendan los reales mecanismos por las cuales se produce, que probablemente sean más bien sociales que individuales.

En este sentido, las actuales políticas públicas en educación, si bien tienden a actuar sobre algunos factores de inequidad (Weinstein, 2001), parecen insuficientes a la hora de recoger el carácter estructural de la violencia ejercida sobre los jóvenes, en particular la que surge desde el propio sistema escolar, en que la actitud más usual es opuesta al reconocimiento: la marginación (García, 1999).

\section{Referencias}

BARUDY, J. (2000). Violencia agresiva y violencia ideológica en la fenomenología humana. En O. Vilches (Ed.) Violencia en la cultura: riesgos y estrategias de intervención. Santiago: Ediciones Sociedad Chilena de Psicología Clínica, pp. 11-29.

BeLLEI, C. (2000). ¿Educación media para todos? Deserción escolar y desigualdad social en Chile. Santiago: Ministerio de Educación, documento de trabajo.

Blanco, R. y Cusato, S. (s.d). Desigualdades educativas en América Latina: todos somos responsables. http:/ / www.uam.es/otros/ rinace / biblioteca / documentos / Blanco.pdf, septiembre 52005.

Bravo, C. (2000). El crudo trasfondo de la violencia escolar. Diario La Nación, edición del 1 de Abril.
Buss, A. (1996). Psicología de la agresión. Buenos Aires: Troquel.

Castillo, C. (1982). Los niños del Perú, clases sociales, ideología y política. Lima: Desco.

Delgado, H. (1938). Psicología general y psicopatología de las tendencias instintivas. Revista de Neuropsiquiatría, 1, 255-353.

ECHeVerría, R. (1996). Ontología del lenguaje. Santiago: Dolmen.

FERNÁNDEZ, I. (1998). Prevención de la violencia y resolución de conflictos. El clima escolar como factor de calidad. Madrid: Narcea S. A. de ediciones.

Ferrán, M. (1996). SPSS para Windows. Programación y análisis estadístico. Madrid: McGraw-Hill.

García, R. (1999). Apoyo a los centros con problemas de violencia. Una tarea de concienciación profesional. Conceptos, 7 , 237-250.

García, E, y Magaz, A. (2000). Modelo Conceptual del TDA-H. http://www.tdah.com/Modelo.html, sep. 52005.

Jones, E. y Gerard, H. (1980). Principios de Psicología Social. México: Limusa.

Leal, F., Sepúlveda, J., Vargas, S., Braniff, M. y IRRAZÁBAL, M. (2003). Desarrollo de un instrumento para la evaluación del resentimiento en población juvenil. Ponencia presentada en el $4^{\circ}$ Congreso Iberoamericano de Evaluación Psicológica, Lima, Perú, 11 y 12 de julio.

León, O. y Montero, I. (1997). Diseño de investigaciones. Introducción a la lógica de la investigación en Psicología y Educación. Madrid: McGraw-Hill.

León, R., Romero, C., Novara, J. y Quesada, E. (1988). Una escala para medir resentimiento. Revista latinoamericana de Psicología, 20, N³, 331-354. 
LERSCH, P. (1971). La estructura de la personalidad. Barcelona: Scientia.

Magendzo, A. (2000). Una aproximación a la relación entre la educación, la disciminación y los derechos humanos. En A. Magendzo y P. Donoso (Eds.), "Cuando a uno lo molestan...:" Un acercamiento a la discriminación en la escuela. Santiago: LOM Ediciones / PIIE.

Maturana, H. (1982). El sentido de lo humano. Santiago: Editorial Universitaria.

MaY, R. (1976). El hombre en busca de sí mismo. Buenos Aires: Central.

Nathan y Harris (1989). Psicopatología y sociedad. México: Trillas.

Nietzsche, F. (1993). La genealogía de la moral. Madrid: M. E. Editores. Ed. original, 1886.

Rodríguez, F.; Gutiérrez, C.; Herrero, F.; Albuerne, F.; Cuesta, M.; Hernández, E.; GÓMEZ, P. Y JiMÉNEZ, A. (2004). Violencia en la enseñanza obligatoria: Alternativas des- de la perspectiva del profesorado. Revista Electrónica de Metodología Aplicada 9(1), 12 27. URL:http://www.psico.uniovi.es/REMA/ v9n1/a2/, sep. 52005.

SCHELLER, M. (1938). El resentimiento en la moral. Buenos Aires: Espasa-Calpe.

Sullivan, H. (1964). La teoría interpersonal de la psiquiatría. Buenos Aires: Psique.

TAттUM, D. (1989). Violent, aggressive and disruptive behavior. En J. Neville (Ed.) Special educational needs review. Vol. 1. Londres: Falmer Press.

Titze, M. (1983). Fundamentos del teleoanálisis adleriano. Barcelona: Herder.

Troncoso, R. (1998). Estudio exploratorio sobre la violencia en jóvenes de enseñanza media. Memoria inédita para optar al Título de Psicólogo, Universidad de Tarapacá, Arica.

Weinstein, J. (2001). Joven y alumno. Desafíos de la Enseñanza Media. Última Década, 15, pp. 99-119. 
Tablas

Tabla 1

Estadísticos descriptivos de los puntajes en la Escala de Resentimiento según grupo de comportamientos agresivos

\begin{tabular}{|l|r|r|r|c|c|}
\hline $\begin{array}{l}\text { Grupo según } \\
\text { comportamientos agresivos }\end{array}$ & $N$ & Media & $\begin{array}{c}\text { Desviación } \\
\text { Estándar }\end{array}$ & Mínimo & Máximo \\
\hline Ausencia & 129 & 10.88 & 6.14 & 2 & 32 \\
\hline Presencia leve & 44 & 12.18 & 5.80 & 3 & 25 \\
\hline Presencia moderada & 82 & 14.30 & 6.14 & 3 & 28 \\
\hline Presencia grave & 15 & 21.93 & 7.54 & 10 & 34 \\
\hline Total & 270 & 12.74 & 6.69 & 2 & 34 \\
\hline
\end{tabular}

Tabla 2:

Análisis de Varianza (ANOVA) de los puntajes de la Escala de Resentimiento según grupo de comportamientos agresivos

\begin{tabular}{|l|c|c|c|c|c|}
\hline $\begin{array}{l}\text { Tipo de } \\
\text { Varianza }\end{array}$ & $\begin{array}{c}\text { Suma } \\
\text { Cuadrática }\end{array}$ & $G l$ & $\begin{array}{c}\text { Media } \\
\text { cuadrática }\end{array}$ & $F$ & Sig. \\
\hline Entre grupos & 1930.494 & 3 & 643.498 & 16.916 & .000 \\
\hline Intra grupos & 10118.872 & 266 & 38.041 & & \\
\hline Total & 12049.367 & 269 & & & \\
\hline
\end{tabular}

Tabla 3:

Comparaciones múltiples para el puntaje en la Escala de Resentimiento entre grupos de comportamientos agresivos, prueba de Scheffé

\begin{tabular}{|l|l|c|c|c|}
\hline \multicolumn{2}{|c|}{$\begin{array}{c}\text { Comparación } \\
\text { (I) Conducta } \\
\text { agresiva }\end{array}$} & $\begin{array}{c}\text { Diferencia } \\
\text { media }(\text { Conducta } \\
\text { agresiva }\end{array}$ & $\begin{array}{c}\text { Errormedia } \\
\text { Estándar }\end{array}$ & Sig. \\
\cline { 1 - 2 } Ausencia & Presencia leve & -1.31 & 1.08 & .689 \\
& Presencia Moderada & $-3.43^{*}$ & .87 & .002 \\
& Presencia grave & $-11.06^{*}$ & 1.68 & .000 \\
\hline Presencia leve & Ausencia & 1.31 & 1.08 & .689 \\
& Presencia Moderada & -2.12 & 1.15 & .337 \\
& Presencia grave & $-9.75^{*}$ & 1.84 & .000 \\
\hline Presencia moderadan & Ausencia & $3.43^{*}$ & .87 & .002 \\
& Presencia leve & 2.12 & 1.15 & .337 \\
& Presencia grave & $-7.63 *$ & 1.73 & .000 \\
\hline Presencia grave & Ausencia & $11.06^{*}$ & 1.68 & .000 \\
& Presencia leve & $9.75^{*}$ & 1.84 & .000 \\
& Presencia Moderada & $7.63 *$ & 1.73 & .000 \\
\hline
\end{tabular}

* La diferencia media es significativa al nivel de 0.05 . 
